PAEDIATRIC LUNG DISEASE

\title{
Trends in deaths from respiratory illness in children in England and Wales from 1968 to 2000
}

\author{
J R Panickar, S R Dodd, R L Smyth, J M Couriel
}

Thorax 2005;60:1035-1038. doi: 10.1136/thx.2005.044750

See end of article for authors' affiliations

\section{Correspondence to:}

Dr J M Couriel, Respiratory Unit, Royal Liverpool Children's Hospital NHS

Trust, Alder Hey, Liverpool L12 2AP, UK; jcouriel@rlc. nhs.uk

Received 17 August 2005 Accepted 21 August 2005 Published Online First 2 September 2005

\begin{abstract}
Background: Childhood mortality has decreased markedly over the last three decades. A study was undertaken to determine trends in deaths from respiratory illness in children in England and Wales.

Methods: Mortality data collected by the Office for National Statistics were analysed. The data included all deaths registered from all causes in children aged between 28 days and 16 years in England and Wales from 1 January 1968 to 31 December 2000. The main outcome measures were overall and age-specific mortality rates due to all respiratory disorders and specific rates for pneumonia, asthma, cystic fibrosis (CF), and bronchiolitis.

Results: In children aged 1-16 years the overall mortality rate (per 100000 children) declined from 49.9 in 1968 to 16.3 in 2000, and rates due to respiratory illness fell from 8.6 to 1.3. The proportion of all deaths caused by respiratory illness in children aged 28 days to 16 years fell from $30.8 \%$ in 1968 to $9.9 \%$ in 2000. In post-neonatal infants (aged 28-364 days), the "all cause" mortality rate fell from 592.8 in 1968 to 176 in 2000 and the rates due to respiratory illness fell from 280 to 22.8 . In 2000, pneumonia, asthma and CF together accounted for $73 \%$ of all respiratory deaths in 1-16 year olds. In this age group, mortality rates per 100000 for pneumonia fell from 4.22 to 0.57 , for asthma from 0.83 to 0.25 , and for CF from 0.66 to 0.12 between 1968 and 2000. Over the same period mortality rates for pneumonia in post-neonatal infants fell from 165 to 6.78 per 100000 and for CF from 4.88 to 0.33 . Bronchiolitis mortality rates per 100000 in post-neonatal infants fell from 21.47 in 1979 to 1.82 in 2000.

Conclusions: Mortality rates due to all respiratory illnesses in children have fallen markedly in the last three decades. This decline has been more rapid than the overall decline in childhood mortality and respiratory diseases are now responsible for a smaller proportion of deaths in children. These data could provide a foundation for assessing the impact on mortality of future health initiatives such as the introduction of a universal pneumococcal vaccination programme in England and Wales.
\end{abstract}

hildhood mortality has decreased markedly in industrialised countries over the last three decades. ${ }^{1-3}$ This fall has been attributed to many different improvements in health care including immunisations, advances in primary, secondary and neonatal care, and better understanding and treatment of childhood illnesses.

Respiratory infections and asthma are among the most common illnesses in childhood, accounting for over a fifth of all general practitioner consultations and 15\% of all hospital admissions for children in the UK. ${ }^{45}$ Most childhood respiratory illnesses are minor and self-limiting, but a minority of episodes of both acute and chronic respiratory disease are fatal. For example, in 1999 respiratory illness accounted for $9.6 \%$ of all deaths in 1-14 year olds in England and Wales, and only injuries and poisonings (23.1\%), malignancies (18.4\%), and central nervous system diseases $(13.0 \%)$ caused more deaths in that year. ${ }^{3}$

All countries need accurate epidemiological information to prioritise, plan, implement and monitor public health interventions. ${ }^{36}$ There have been several studies of childhood deaths from individual respiratory illnesses such as asthma ${ }^{7-9}$ and cystic fibrosis, ${ }^{10}{ }^{11}$ but we are not aware of a detailed analysis of the trends in all the respiratory causes of deaths in children in England and Wales over a prolonged period. The aim of this study was to investigate these trends and to analyse the changing patterns of deaths from the individual diseases which are responsible for most respiratory deaths in children-namely, pneumonia, asthma, cystic fibrosis and bronchiolitis.

\section{METHODS}

The data were provided by the Office for National Statistics (ONS). They consisted of all deaths registered from all causes in children aged between 28 days and 16 years in England and Wales from 1 January 1968 to 31 December 2000. The data included year of death, cause of death under the International Classification of Disease (ICD), age at death, and sex. ICD eighth revision (ICD-8) codes were used from 1968 to 1978 and ICD ninth revision (ICD-9) codes from 1979 to 2000 . We defined deaths from a respiratory cause as deaths recorded in ICD categories 460-519.9 combined with deaths due to cystic fibrosis (273.0 in ICD 8, 277.0-277.01 in ICD 9), pulmonary tuberculosis (011-012.9 and 019.0 in both ICD 8 and 9), whooping cough (pertussis) (033.0-033.9), and congenital anomalies of the respiratory system (748.0-748.9).

The data were analysed in four age groups: post-neonatal infants (age 28-364 days), 1-5 years, 6-10 years, and 1116 years. Mortality rates were expressed per 100000 population using the mid-year age-specific populations estimated by the ONS. Neonatal deaths (deaths of infants aged less than 28 days) were excluded for two reasons: (1) because the respiratory causes of deaths are very different in neonates ${ }^{4}$ and (2) since the introduction of a neonatal death certificate in 1986 it has not been possible to assign an underlying cause of death in this age group. ${ }^{12}$

We calculated overall mortality rates, age-specific mortality rates due to all respiratory causes, and specific rates for pneumonia, asthma, cystic fibrosis, and the post-neonatal infant mortality rates for bronchiolitis. Bronchiolitis mortality 


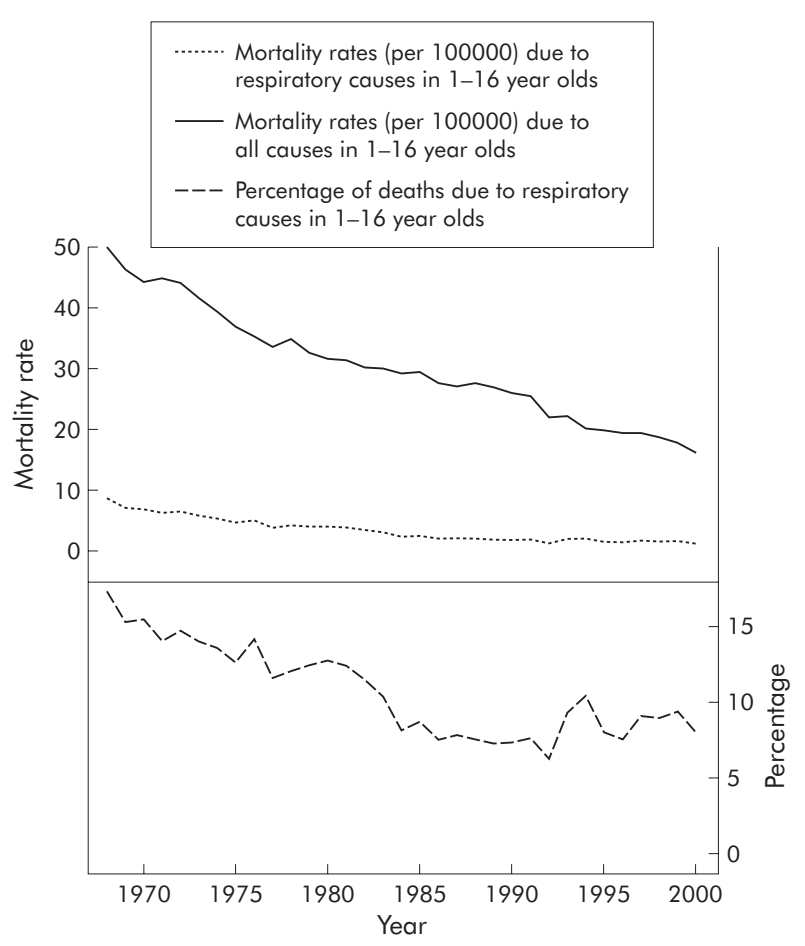

Figure 1 Mortality rates and percentage of deaths due to respiratory causes in children aged 1-16 years from 1968 to 2000.

rates were analysed from 1979 to 2000 as a separate ICD coding for acute bronchiolitis was used from ICD-9. In ICD-8 acute bronchitis and bronchiolitis were coded together (466).

Stata version 8 and SPSS version 11.0 were used for statistical analysis.

\section{RESULTS}

The mortality rates from all causes for children aged 116 years were 49.9 per 100000 in 1968, 29.6 in 1985, and 16.3 in 2000 . The mortality rates from respiratory illness in this age group were 8.6, 2.6, and 1.3 per 100000 in 1968, 1985 and 2000, respectively (fig 1). The proportion of deaths due to respiratory causes in children aged $1-16$ years fell from $17.3 \%$ in 1968 to $8.8 \%$ in 1985 and $8.0 \%$ in 2000 (fig 1).

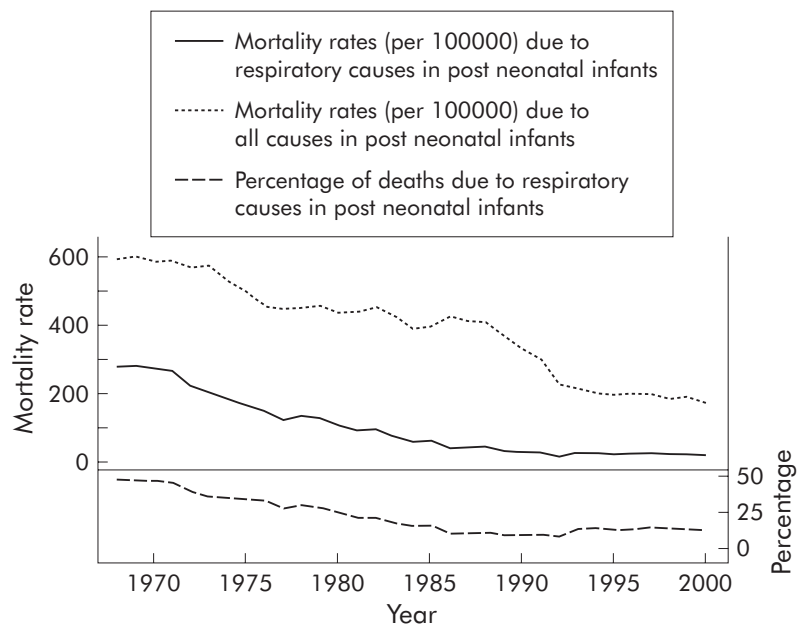

Figure 2 Mortality rates and percentage of deaths due to respiratory causes in post-neonatal infants (aged between 28 and 364 days) from 1968 to 2000.

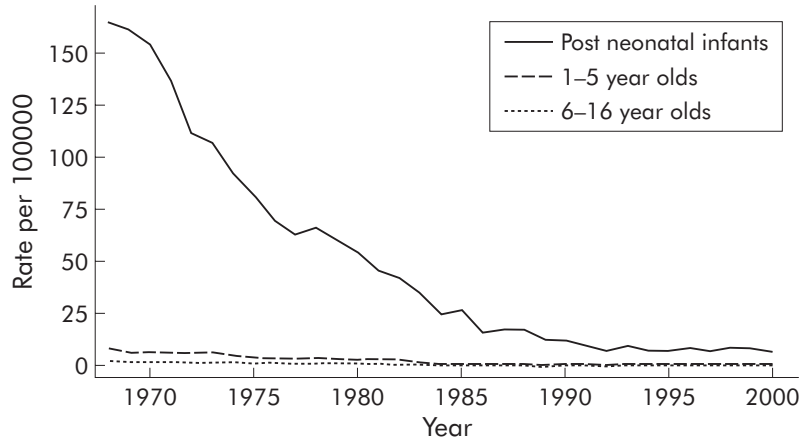

Figure 3 Mortality rates due to pneumonia from 1968 to 2000.

By 2000, pneumonia (43\%), asthma (20\%), and cystic fibrosis ( $10 \%$ ) accounted for $73 \%$ of the 138 deaths due to respiratory illness in $1-16$ year olds.

Far higher mortality rates were seen in post-neonatal infants (age 28-364 days). In this group the overall mortality rates fell from 592.8 in 1968 to 397.6 in 1985 and to 176 in 2000. The mortality rates from respiratory illness in these infants were 280, 65.1, and 22.8 in 1968, 1985 and 2000, respectively. The percentage of deaths due to respiratory causes in this age group fell from 47.2 in 1968 to 16.4 in 1985 and to 13.0 in 2000 (fig 2).

Mortality rates due to childhood pneumonia fell in all age groups (fig 3). The most striking change was in post-neonatal infants where the mortality rate fell from 165 per 100000 in 1968 to 27 in 1985 and 6.78 in 2000, a fall of $96 \%$ in this age group. The absolute numbers of deaths attributed to pneumonia in children aged 28 days to 16 years were 1855 in 1968, 224 in 1985, and 101 in 2000, a fall of 95\% over 32 years.

Figure 4 shows the trends for deaths from asthma in three age groups. Age-specific mortality rates due to asthma have declined dramatically. Between 1968 and 2000 mortality rates due to asthma decreased by $78 \%$ (from 0.58 to 0.13 ) in $1-5$ year olds, $57 \%$ (from 0.53 to 0.23 ) in $6-10$ year olds, and $73 \%$ (from 1.38 to 0.37 ) in $11-16$ year olds. The rates were consistently higher in children aged 11-16 years than in younger children. In 1968, 99 children aged 1-16 years died from asthma compared with 50 and 27 deaths in 1985 and 2000, respectively.

Deaths from cystic fibrosis fell in all age groups since the late 1960s, with the most marked decline in post-neonatal infants (fig 5). The trends in mortality rates were very similar in the 6-10 year and the 11-16 year groups, so these are not shown separately. The rise in mortality rates in 6-16 year olds in the late 1970s and early 1980s was accompanied by a fall in mortality rates in infants and children aged $1-5$ years. In

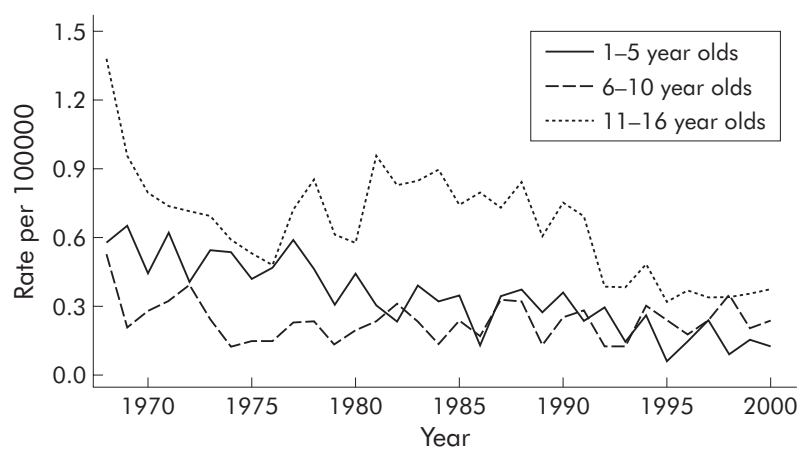

Figure 4 Mortality rates due to asthma from 1968 to 2000. 


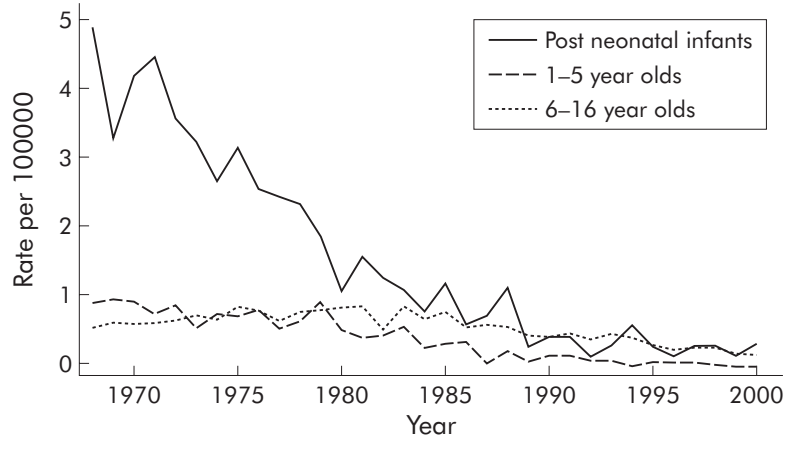

Figure 5 Mortality rates due to cystic fibrosis from 1968 to 2000.

1968,118 deaths were attributed to cystic fibrosis in children aged 28 days to 16 years compared with 75 in 1985 and 15 in 2000 .

Severe acute viral bronchiolitis is seen almost exclusively in infants aged between 1 month and 1 year. The mortality rate due to bronchiolitis in post-neonatal infants fell from 21.47 in 1979 to 1.82 in 2000 (fig 6), and the absolute number of deaths fell from 137 to 11 per annum in the same period.

Mean (SD) male:female mortality rate ratios between 1968 and 2000 were $1.24(0.17)$ for pneumonia, 1.41 (0.48) for asthma, and $0.82(0.28)$ for cystic fibrosis for children aged 28 days to 16 years, and 1.77 (1.25) for bronchiolitis in postneonatal infants. There was no significant trend for male:female mortality rate ratios for pneumonia, asthma, cystic fibrosis, or bronchiolitis during the study period.

\section{DISCUSSION}

We have shown that, as in all industrialised countries, mortality rates in children have fallen dramatically in England and Wales in the last 32 years. The mortality rates due to respiratory illness have fallen more steeply than the overall rates, and a much smaller proportion of childhood deaths is now due to respiratory disease than in the past. The greatest improvements have been in infants and young children. Between 1968 and 2000, post-neonatal mortality rates from all causes decreased by $70 \%$, while the rates due to respiratory deaths fell by $92 \%$. In 1968 almost half the deaths in post-neonatal infants were attributed to respiratory disease but, by 2000 , this proportion had fallen to $13 \%$. In 1-16 year old children mortality rates for total deaths and respiratory deaths fell by $67 \%$ and $85 \%$, respectively, between 1968 and 2000, and the percentage of deaths from respiratory disease fell from 17.3 in 1968 to 8.8 in 1985 and to 8.0 in 2000.

There are some limitations to this study. A significant proportion of infants and children who die from pneumonia or bronchiolitis have a serious underlying condition such as congenital anomalies of the cardiorespiratory system or CNS, a chromosomal abnormality, or an immunodeficiency. The ONS does not collect or provide data about these important co-existent conditions which significantly predispose children to death from a respiratory illness. We therefore cannot comment on their contribution to the trends we report. During the period we analysed there were several changes in data collection by the ONS and its predecessors. Between 1984 and 1992 there were changes in the selection of the underlying cause of death from the death certificate. From 1993 automated coding was used to assign the cause of death. It has been suggested that changes in the mortality rates for pneumonia from 1993 may have been influenced by changes in data collection, but it is difficult to quantify these effects accurately. ${ }^{12}{ }^{13}$ However, our data do not show any

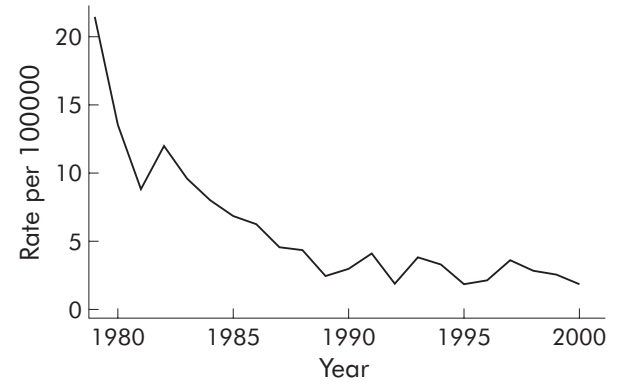

Figure 6 Mortality rates (per 100000 ) due to bronchiolitis in postneonatal infants from 1979 to 2000 .

sudden alteration in mortality from pneumonia in children at the time of these changes.

Changes in ICD coding can also affect mortality rates for asthma. ${ }^{14}$ For example, in 1979, with the introduction of the ninth revision of the ICD (ICD-9), deaths due to asthma with a mention of bronchitis were recorded under asthma unspecified (493.9) rather than bronchitis (490). This change in classification resulted in an apparent increase in the numbers of deaths from asthma, particularly in adults where there may be confusion with other diseases such as chronic obstructive pulmonary disease. Stewart and Nunn ${ }^{15}$ reported that the change from ICD-8 to ICD-9 coding caused an apparent $28 \%$ increase in asthma mortality in the total population, although in younger age groups the effect was smaller. A survey of deaths from asthma in England showed death certification was more accurate in younger adults than in the elderly. ${ }^{16}$ Sears et al ${ }^{17}$ showed that in New Zealand the accuracy of certification for asthma deaths was 100\% in subjects under 35 years compared with only $50 \%$ in patients over 70 years. However, there is little evidence to suggest that changes in diagnostic labelling by clinicians or in ICD coding explains the changes in the mortality rates from asthma for children we describe.

Because pneumonia, asthma, cystic fibrosis, and bronchiolitis together accounted for most of the deaths due to respiratory illness in children aged between 28 days and 16 years in the period studied, we examined these conditions in more detail.

Mortality rates due to childhood pneumonia have fallen in all age groups. The rates fell sharply between 1968 and the mid 1980s and have since levelled out. The fall was greatest in post-neonatal infants but, even now, most childhood deaths from pneumonia occur in the first year of life. In 2000, $40 \%$ of deaths due to pneumonia in children aged 28 days to 16 years occurred in post-neonatal infants. Our data cannot explain why the number of deaths in children from pneumonia has fallen, but it is probable that improvements in primary, secondary and specialist care, in the use of antimicrobial agents, and in immunisations have all contributed to this reduction. For example, Mulholland et al ${ }^{18}$ reported that $\mathrm{HiB}$ (Haemophilus influenzae $\mathrm{B}$ ) conjugate vaccine, which was introduced into the UK in 1992, is 95\% effective in preventing invasive $\mathrm{HiB}$ disease, $100 \%$ effective in preventing $\mathrm{HiB}$ pneumonia, and effective in preventing $21 \%$ of all radiologically defined pneumonia. The high levels of protection against pertussis and measles provided by immunisation are also likely to have affected mortality. It will be important to study the effect of the proposed programme of pneumococcal vaccination in children on mortality from pneumonia in this population. These data could provide a valuable baseline to assess the impact of new public health clinical measures which may be introduced in the near future. For example, a universal immunisation programme against pneumococcal infection has been shown to be highly 
effective in preventing invasive pneumococcal disease, with a reduction in the incidence of radiologically confirmed pneumonia in children of about $25 \% .{ }^{19}$

We found that a decline in asthma mortality rates until the mid 1970s was followed by a rise in the 1980s, a further fall in the early 1990s, and a levelling out since the mid 1990s. This pattern differs somewhat from that in other countries. In the United States, in children aged 0-17 years the asthma mortality rate increased by an average of 3.4\% per year from 1980 to $1998 .{ }^{8}$ In Australia and Germany, asthma related deaths in childhood showed an increase between the mid 1970s and mid 1980s. ${ }^{20}$ Campbell et al reported an irregular downward trend in 5-14 year olds from 1983 tol995 in England and Wales with an average fall of about $6 \%$ a year. ${ }^{9}$

The survival of children and adults with cystic fibrosis has improved dramatically in the last 30 years. ${ }^{21}$ In 1968 there were 118 deaths from cystic fibrosis in children but, by the year 2000, there were only 15 . The small increase in deaths in 6-16 year olds in the 1970-1980s occurred when there was a steep fall in post-neonatal mortality rates and mortality rates in 1-5 year olds, which suggests that this reflects survival to a later age. This improved survival is due to multiple factors including earlier diagnosis and more intensive and effective treatment. It is possible that the figures may have been influenced by changes in coding and death certification and more accurate diagnosis. In the past, the recognition of deaths from cystic fibrosis may have been incomplete and mortality rates may have been underestimated. We cannot assess the influence on mortality from cystic fibrosis of factors which can alter the prevalence of the disease such as prenatal diagnosis with therapeutic terminations, or of neonatal screening which became available during the period of our study.

Acute viral bronchiolitis is an important cause of hospital admissions and need for intensive care in infants. Deaths due to bronchiolitis in post-neonatal infants fell by over $92 \%$ between 1979 and 2000. During this time there was no change in ICD coding and no evidence of any change in the prevalence of bronchiolitis. It is probable that the reduction in the number of deaths reflects improvements in paediatric care, particularly in paediatric intensive care.

In conclusion, we have observed a marked and progressive fall in deaths in childhood from respiratory disease. This decline has been more rapid than that seen for childhood deaths from all causes, so that today a smaller proportion of childhood deaths are the result of respiratory illness. The most significant decline was in deaths from pneumonia in the first year of life.

\section{ACKNOWLEDGEMENTS}

The authors thank Moira Mickelburgh of the Office of National Statistics for providing the data.

\section{Authors' affiliations}

J R Panickar, J M Couriel, Respiratory Unit, Royal Liverpool Children's Hospital NHS Trust, Alder Hey, Liverpool L12 2AP, UK

S R Dodd, Centre for Medical Statistics and Health Evaluation, University of Liverpool, Liverpool L69 3GS, UK

R L Smyth, Division of Child Health, University of Liverpool, Royal Liverpool Children's Hospital NHS Trust, Alder Hey, Liverpool L12 2AP, UK

Funding: Respiratory Unit, Royal Liverpool Children's NHS Trust Competing interests: none declared.

The work was independent of the funders.

\section{REFERENCES}

1 UNICEF. The state of the world's children, 2003. http://www.unicef.org/ sowc03/tables/table5.html (accessed 28 January 2005).

2 UNICEF. Child mortality statistics. http://www.childinfo.org/cmr/revis/ $\mathrm{db} 2 . \mathrm{htm}$ (accessed 28 January 2005).

3 Logan S. Epidemiology of child health. In: Mclntosh N, Helms P, Smyth R, eds. Forfar and Arneil's textbook of paediatrics. 6th ed. Edinburgh: Churchill Livingstone, 2003.

4 Christopher A, Crowley S. Burden of respiratory disease in childhood. Airways J 2004;2:104-7.

5 Couriel J. Assessment of the child with recurrent chest infections. Br Med Bull 2002;61:115-32.

6 Black RE, Morris SS, Bryce J. Where and why are 10 million children dying every year? Lancet 2003;361:2226-34.

7 Burney P. Asthma deaths in England and Wales 1931-85: evidence for a true increase in asthma mortality. J Epidemiol Community Health 1988:42:316-20.

8 Akinbami L, Schoendorf KC. Trends in childhood asthma: prevalence, health care utilization and mortality. Pediatrics 2002;110:315-22.

9 Campbell MJ, Cogman GR, Holgate ST, et al. Age specific trends in asthma mortality in England and Wales, 1983-95: results of an observational study. BMJ 1997;314:1439-41.

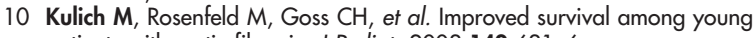
patients with cystic fibrosis. J Pediatr 2003;142:631-6.

11 Halliburton CS, Mannino DM, Olney RS. Cystic fibrosis deaths in the United States from 1979 through 1991. An analysis using multiple-cause mortality data. Arch Pediatr Adolesc Med 1996;150:1181-5.

12 Office for National Statistics. Mortality statistics: cause. Review of the Registrar General on deaths by cause, sex and age in England and Wales, 2001. DH2 no 28. Office for National Statistics, 2002.

13 Rooney C, Devis T. Mortality trends by cause of death in England and Wales 1980-94: the impact of introducing automated coding and related changes in 1993. Popul Trends 1996;86:29-35.

14 Jannsen $F$, Kunst $A E$. ICD coding changes and discontinuities in trends in cause-specific mortality in six European countries, 1950-99. Bull WHO 2004;82:904-13.

15 Stewart CJ, Nunn AJ. Are asthma mortality rates changing? $\mathrm{Br} J$ Dis Chest 1985;79:229-234.

16 BTA Research Committee. Accuracy of death certificates in bronchial asthma. Thorax 1984;39:505-9.

17 Sears MR, Rea HH, de Boer G, et al. Accuracy of certification of deaths due to asthma. A national study. Am J Epidemiol 1986;124:1004-11.

18 Mulholland K, Hilton S, Adegbola R, et al. Randomised trial of Haemophilus influenzae type-b tetanus protein conjugate vaccine for prevention of pneumonia and meningitis in Gambian infants. Lancet 1997;349:1191-7.

19 O'Brien KL, Santosham M. Potential impact of conjugate pneumococcal vaccine on pediatric pneumococcal diseases. Am J Epidemiol 2004; 159:634-44.

20 Jackson R, Sears MR, Beaglehole R, et al. International trends in asthma mortality: 1970 to 1985. Chest 1988;94:914-8.

21 Dodge JA, Morison S, Lewis PA, et al. Incidence, population and survival of cystic fibrosis in the UK, 1968-95. Arch Dis Child 1997;77:493-6. 